\title{
14 Years of Daily Timing Observations of the Vela Pulsar
}

\author{
P M McCulloch \\ Physics Department, University of Tasmania, GPO Box 252c Hobart \\ 7001, Australia
}

\begin{abstract}
Daily timing observations of the Vela pulsar have been made over the last 14 years. During this time there has been seven large period jumps, or glitches, four of these have been observed as they occurred. We examine the time scale of these glitches and several other events which appear to be mini-glitches.
\end{abstract}

\section{Introduction}

A $14 \mathrm{~m}$ antenna at the University of Tasmania has been devoted to observations of the Vela pulsar since October 1981. Prior to 1987 the antenna had an equatorial mount which limited daily observations to 5 hours. These early observations were made by recording all four Stokes parameters at $635 \mathrm{MHz}$. In 1988 the antenna was remounted and relocated at the Mt Pleasant Observatory, with the result that Vela could be tracked for 18 hours each day, and the receiving equipment upgraded to provide simultaneous observations at 635 and $950 \mathrm{MHz}$.

\section{Observations}

The data was folded at the pulsar period to produce an integrated pulse profile every two minutes which was then compared with a standard pulse template to give a pulse arrival time. These local arrival times were reduced to barycentric arrival times with the aid of the JPL DE200 ephemeris. During the interval 1981-87 the period of the Vela pulsar decreased abruptly, glitched, on three occasions. While these glitches were not observed directly, on each occasion data was recorded a few hours after the jump, McCulloch et al 1983, 1987, giving the first insight into the details of the glitch phenomenon. On Christmas Day 1988 another glitch occurred, this time while we were recording data, McCulloch et al 1990. Since that time three more large glitches have occurred, each of them while we were observing.

\section{Results}

Since 1981 we have accumulated over one million arrival time measurements of the Vela pulsar giving us a much more extensive data base than exists for any other pulsar. Arrival time data over successive interval of eight days have been used to calculate the pulse period as a function of epoch, figure 1a. The pulse 

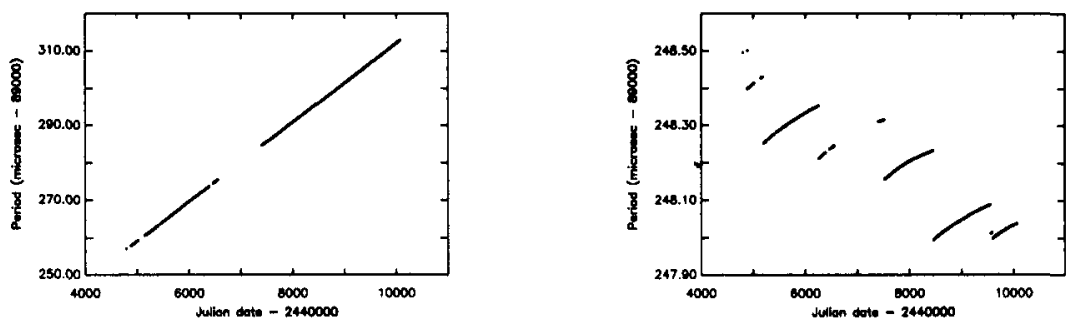

Figure 1. a). Period as a function of epoch. b). same as a) but with a linear trend removed.
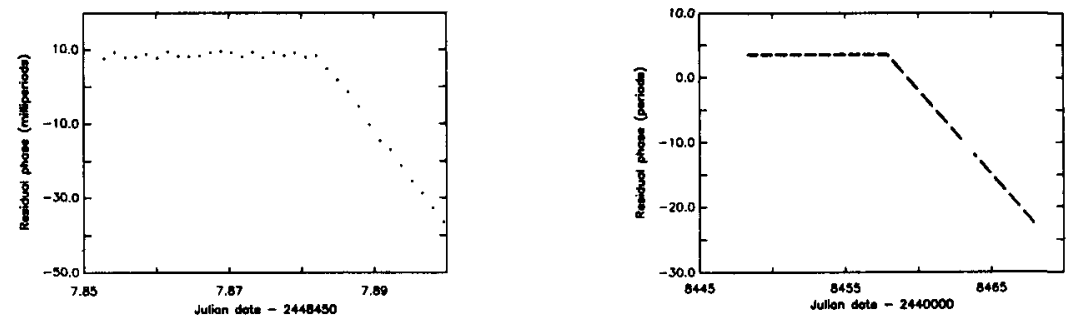

Figure 2. Glitch of July 1991 at low time resolution (left) and showing individual 2 minute data points (right).

period increased almost monotonically by about $55 \mu$ sec over this time interval, however when a linear trend is removed from this data, figure $1 \mathrm{~b}$, we find that the period increase is not linear but slows down and also shows a number of abrupt period decreases of a few tenths of a microsecond or parts in $10^{6}$.

Figure 2 shows the glitch of July 1991 at two different time resolutions, the high resolution plot shows the individual arrival times separated by about 2 minutes and inspection indicates the the period change was close to instantaneous and occurred within about a 2 minute time interval.

Following this event we modified the equipment to improve the time resolution by de-dispersing eight contiguous channels, thereby increasing the signal to noise ratio and allowing us to detect single pulses. This system was operated for two years by recording single pulses on a magnetic disk. The disk had sufficient capacity for three days data and was over written unless we detected a glitch.

In 1994 two glitches were observed within the space of a few months, Flanagan, 1994a, b, McCulloch 1994, these were both relatively small events, however the single pulse equipment was operating and we recorded data from the first, larger event. This is shown in figure 3 , where the left frame shows the glitch at low resolution and the right frame shows individual arrival times made by averaging over intervals of 30 seconds.

The signal to noise ratio is clearly much worse than for the the 1991 event, partly because this glitch was a factor of 5 smaller and partly because of our shorter integration time. However it is apparent that the glitch occurred on a 

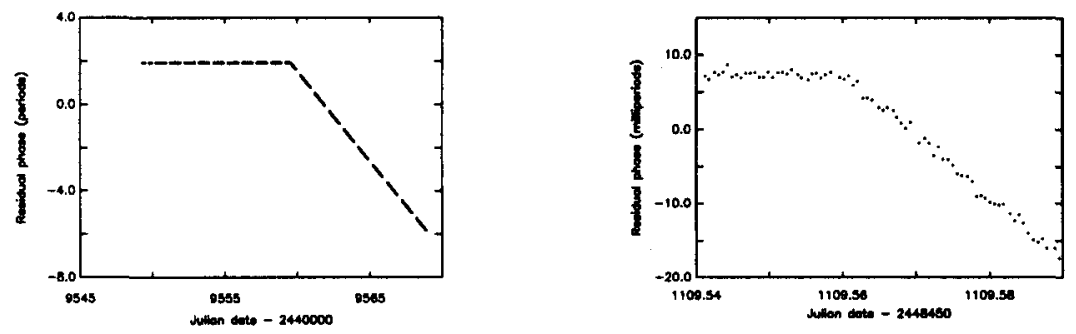

Figure 3. Glitch of July 1994 at low resolution (left) and with 30 second resolution (right).
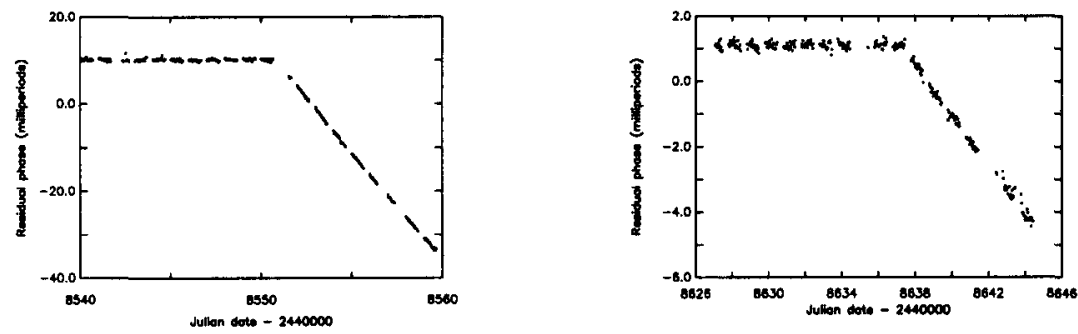

Figure 4. Two small period jumps which have the same characteristics as large glitches.

short time scale of less than a few minutes and the data are consistent with an instantaneous jump in period.

As well as these large glitches, timing observations of the Vela pulsar show a wealth of activity generally described as timing noise, eg Boynton et al. 1972, Cordes and Downs 1985, which has been described in terms of a number of random processes. The changes in pulse arrival time accompanying this timing noise appear to occur relatively slowly compared to the glitch activity, sometimes taking hours or days to settle to a new period, indicating that this is a different phenomena. Detailed examination of our data during the interval July 1991 to June 1994 has revealed two events which are more characteristic of glitches than timing noise. The period change associated with these events are several orders of magnitude smaller than normal glitches but otherwise they appear identical to the large glitches as shown in figure 4.

The accumulated phase change over several days for these events are only tens of milliperiods rather than tens of periods, but the events appear to be instantaneous changes in period, indicating that glitches in the Vela pulsar can range in amplitude over many orders of magnitude. Without continuous arrival time measurements these events would almost certainly be classified as timing noise.

The data following the 1988 and subsequent large glitches has been analysed using an empirical timing model with three time scales, about a day, a few days and a third much longer recovery time. In each case data from 30 days prior to 
the glitch was used to determine a slow down model $\nu_{0}$, with the pulse frequency $\nu$ at time $\mathrm{t}$ after the glitch being given by

$$
\nu=\nu_{0}+\Delta \nu_{0}+\Delta \nu_{1} e^{-\frac{t}{\tau_{1}}}+\Delta \nu_{2} e^{-\frac{t}{\tau_{2}}}+\Delta \nu_{3} e^{-\frac{t}{\tau_{3}}}
$$

The parameters for this fit, the glitch epochs and sizes are given in table 1 .

Note that the first two glitches are very similar, they were both large amplitude events with very similar recovery patterns. The most significant difference between them is the much larger change in frequency derivative for the second glitch which arises because of the larger amplitude of the rapid recovery term. This appears to be real although the data for the first glitch was of somewhat poorer quality making it difficult to get very accurate arrival time close to the glitch. The first glitch of 1994 is somewhat different, it was smaller in magnitude and was not accompanied by any significant change, or recovery, in frequency derivative. The small interval between this and the next event makes it impossible to look for long term recovery effects. The second glitch of 1994 was even smaller in magnitude but did involve a change and subsequent recovery in frequency derivative similar to those of the earlier glitches.

Table 1. Glitch parameters from 1988 to 1996

$\begin{array}{ccccc}\text { Date } & \text { December 1988 } & \text { July 1991 } & \text { July 1994 } & \text { August 1994 } \\ \text { Epoch JD-2440000 } & 7520.3036 & 8457.8823 & 9559.560 & 9591.658 \\ \Delta \nu_{0}(\mu \mathrm{Hz}) & 13.297 & 27.079 & 9.634 & 2.132 \\ \Delta \nu_{1}(\mu \mathrm{Hz}) & 0.092 & 0.255 & 0.00 & 0.024 \\ \tau_{1} \text { (days) } & 0.73 & 0.56 & - & 1.59 \\ \Delta \nu_{2}(\mu \mathrm{Hz}) & 0.083 & 0.169 & - & 0.027 \\ \tau_{2} \text { (days) } & 6.97 & 5.94 & - & 15 \\ \Delta \nu_{3}(\mu \mathrm{Hz}) & 6.74 & 2.84 & - & - \\ \tau_{3} \text { (days) } & 707 & 254 & - & - \\ \frac{\Delta \nu}{\nu} 10^{-6} & 1.8 & 2.71 & 0.86 & 0.19 \\ \frac{\Delta \dot{\nu}}{\dot{\nu}} & 0.086 & 0.39 & 0.0 & 0.02\end{array}$

\section{References}

Boynton P E et al, 1972, ApJ, 175, 217

Cordes J M \& Downs G S, 1985, ApJS, 59, 343

Flanagan C, 1994, IAU Circular 6038

Flanagan C, 1994, IAU Circular 6064

McCulloch P M, Hamilton P A, Royle G W R \& Manchester R N,1983, Nature 302,319

McCulloch P M, Klekociuk A R, Hamilton P A \& Royle G W R, 1987, Aust. J. Phys. 40, 725

McCulloch P M, Hamilton P A, McConnell D \& King E A, 1990, Nature, 346, 822

McCulloch P M, 1994, IAU Circular 6038 